\title{
Japanese study to organize proper lifestyle modifications for metabolic syndrome (J-STOP- MetS): Design and method
}

\author{
Masanori Munakata' \\ Hiroki Honma ${ }^{2}$ \\ Mitugu Akasi ${ }^{3}$ \\ Takaharu Araki ${ }^{4}$ \\ Takahiko Kawamura ${ }^{5}$ \\ Masashi Kubota ${ }^{6}$ \\ Tomoko Yokokawa ${ }^{7}$ \\ Akira Maruhashi ${ }^{8}$ \\ Toshihiro Toyonaga ${ }^{9}$ \\ On behalf of the J-STOP- \\ MetS Study Group \\ All authors belong to Preventive \\ Medical Centers of Rosai Hospital \\ Groups in Japan; 'Tohoku Rosai \\ Hospital, Sendai, Japan; ${ }^{2}$ Iwamizawa \\ Rosai Hospital, Iwamizawa, Japan; \\ ${ }^{3}$ Kantou Rosai Hospital, Kawasaki, \\ Japan; ${ }^{4}$ Tokyo Rosai Hospital, Tokyo, \\ Japan; ${ }^{5}$ Cyubu Rosai Hospital, Nagoya, \\ Japan; ${ }^{6}$ Oosaka Rosai Hospital, \\ Sakai, Japan; ${ }^{7}$ Kansai Rosai Hospital, \\ Amagasaki, Japan; ${ }^{8}$ Chugoku Rosai \\ Hospital, Kure, Japan; ' $\mathrm{K}$ yusyu Rosai \\ Hospital, Kokura, Japan
}

Correspondence: Masanori Munakata Preventive Medical Center,Tohoku Rosai Hospital, 3-2I Dainohara 4,Aobaku, Sendai 98I-8563, Japan

Tel +8 I 022275 I I I I (ext. 22 I4)

Fax +8I 0222736606

Email: munakata.@tohokuh.rofuku.go.jp

\begin{abstract}
Prevalence of the metabolic syndrome is now a very serious health problem in Japan and a public preventive strategy is essential to reduce morbidity. A systematic interventional strategy for the metabolic syndrome remains to be established. In order to address this issue, a multi-center study; Japanese Study to Organize Proper lifestyle modification for the metabolic syndrome (J-STOP-MetS), has been established by nine preventive medical centers among Rosai hospital groups. This study comprises a cross-sectional study (J-STOP-MetS 1) and a prospective randomized control study (J-STOP-MetS 2). J-STOP-MetS 1 examines the causes of the metabolic syndrome by means of a questionnaire in a large cohort of patients with the metabolic syndrome and control subjects matched for age and sex. J-STOP-MetS 2 examines the hypothesis that guidance on lifestyle modifications will help at risk patients to reduce abdominal fat and cardiovascular risk factors. The metabolic syndrome patients are randomly assigned either to a single visit to a guidance group or multiple visits every two months. The individualized guidance is provided by the coordination of physician, trained nurse, dietician and exercise trainer. Several parameters are measured before and six months after the first guidance session, including, body weight, waist circumference, blood pressure, several blood markers and arterial stiffness. The J-STOP-MetS is the first large-scale clinical study of the metabolic syndrome in Japan and should provide important evidence for the practical management of the metabolic syndrome.
\end{abstract}

Keywords: metabolic syndrome, J-STOP-MetS, hypertension, diabetes, dyslipidemia

\section{Introduction}

The prevalence of obesity in Japan has increased since the 1980s and is observed in all age groups of men and in older groups of women (Yoshiike et al 2002; Sakamoto 2006). Obesity is strongly associated with established cardiovascular risk factors such as hypertension, hyperglycemia and dyslipidemia. Metabolic syndrome has recently received great interest, as patients with this syndrome are at high risk of developing coronary artery disease (Baltali et al 2003; Kip et al 2004). This syndrome is characterized by a group of metabolic risk factors occurring in one person. These include abdominal fat accumulation, insulin resistance, high blood pressure and abnormalities in lipid and glucose metabolism. A recent Japanese governmental survey has shown that $45.6 \%$ of men and $16.7 \%$ of women aged between 20 and 74 years have been diagnosed with the metabolic syndrome or have a preliminary stage of this condition (see http://www.mhlw.go.jp/houdou/2006/05/ h0508-1a.html). This national increase in obesity, especially in men, is of great concern because it will result in a higher morbidity and mortality rate, due to atherosclerotic cardiovascular diseases and, as a consequence of this, an increase in healthcare costs is inevitable. 
The imbalance between energy intake and energy expenditure has led to increasing obesity. However, determinants of eating behavior or physical activity are very complex. Recent studies have shown that stress at work is an important risk factor for the metabolic syndrome (Chandola et al 2006), and that shift work may increase metabolic risk factors of cardiovascular disease (Karlsson et al 2001). These data suggest that unhealthy behavior that predisposes the metabolic syndrome is linked to numerous environmental factors.

A public prevention strategy is essential to reduce the morbidity of the metabolic syndrome. To achieve this, we must clarify factors which could cause the metabolic syndrome, since in highly developed country like Japan, there may be several unknown environmental risk factors. Furthermore, effective guidelines for lifestyle modifications in the metabolic syndrome remain to be established. To address these issues, we have established a multi-center study; Japanese Study To Organize Proper lifestyle modification for the metabolic syndrome (J-STOP-MetS).

\section{Methods}

\section{J-STOP-MetS I}

The J-STOP-MetS 1 is a cross-sectional study that examines the possible causes of the metabolic syndrome by means of the questionnaire in a large cohort of metabolic syndrome patients and a control group of matched healthy subjects at least 1000 subjects in each group. Diagnosis of the metabolic syndrome in men is based on new Japanese criteria (CEDSMSJ 2005) that includes waist circumference $85 \mathrm{~cm}$ or over with at least two cardiovascular risk factors, among which are high blood pressure (systolic blood pressure $\geq 130$ $\mathrm{mmHg}$ or diastolic blood pressure $\geq 85 \mathrm{mmHg}$ ), hyperglycemia (fasting blood sugar $\geq 110 \mathrm{mg} / \mathrm{dL}$ ) and dyslipidemia (triglyceride $\geq 150 \mathrm{mg} / \mathrm{dL}$, or $\mathrm{HDL}<40 \mathrm{mg} / \mathrm{dL}$ ). Use of antihypertensive, antidiabetic or antidyslipidemic agents is considered as an indication for each respective risk factor. For women, the diagnostic cutoff value for waist circumference was set at $90 \mathrm{~cm}$ in Japanese criteria but we have used $80 \mathrm{~cm}$ in this study, because recent studies have consistently shown that most suitable cutoff level exists at around $80 \mathrm{~cm}$ (Miyawaki et al 2005; Hara et al 2006). This is consistent with Asian criteria (Alberti et al 2005). Waist circumference was measured at the umbilical level in the standing position by a trained staff member. More than one hundred cases and similar number of age and sex matched controls will be at least collected at each center.

Exclusion criteria are severe obesity (body mass index $\geq 35 \mathrm{~kg} / \mathrm{m}^{2}$ ), diabetic patients treated with insulin, uncontrolled diabetes (fasting blood glucose $\geq 160 \mathrm{mg} / \mathrm{dL}$ or $\mathrm{HbAl} 1 \mathrm{c} \geq 8.0 \%$ ), renal failure (plasma creatinine concentration $\geq 1.5 \mathrm{mg} / \mathrm{dL}$ ), and previous history of cardiovascular diseases, cancer or other serious diseases. After written informed consent is obtained, each subject is given a detailed questionnaire asking questions about their work, degree of physical activity in daily life, past and present illness, present medications, beverages, dietary and exercise habits, stress at work and at home, type A behavior and depression and life style characteristics during childhood. Type A behavior was assessed by Maeda checklist (Maeda 1985) and the degree of masked depression was assessed by a self-rating questionnaire for depression (Abe et al 1972). The validity, reliability and clinical usefulness of those questionnaire have been reported previously (Munakata et al 1998). The questionnaire was reviewed by all physicians, nurses, dieticians and exercise trainers involved in this study beforehand.

These factors are compared between patients with the metabolic syndrome and control subjects. The control subjects must have normal waist circumference $(<90 \mathrm{~cm}$ for men and $<80 \mathrm{~cm}$ for women) with normal blood pressure $(140 / 90 \mathrm{mmHg}$ ), normoglycemia (fasting blood sugar $<110$ $\mathrm{mg} / \mathrm{dL}$ ) and normolipidemia (triglyceride $<150 \mathrm{mg} / \mathrm{dL}$ and $\mathrm{HDL} \geq 40 \mathrm{mg} / \mathrm{dL}$ ). The control subjects must also not be taking antihypertensive, antidiabetic or antidyslipidemic agents. Metabolic syndrome patients are recruited from the patients found in outpatient clinics of hypertension, diabetes or dyslipidemia or from those found in annual health-check up of several companies. Age- and sex-matched control was recruited from the subjects and fulfilled the normal criteria in annual health-check up.

\section{J-STOP-MetS 2 \\ Outline of the study}

The J-STOP-MetS 2 is a prospective randomized study which examines the hypothesis that guidance for life style modification will help at risk patients to reduce abdominal fat and cardiovascular risk factors (Figure 1). In Japan, such guidance has been usually offered as a one-off, associated with annual health check. However, this single guidance approach has not yielded good results, as indicated by the increasing incidence of obesity. Furthermore, the guidance offered has never been standardized. In this study, we examine whether the results can be improved by increasing the frequency of guidance and offering guidance which is highly standardized and individualized.

In our preliminary data, mean body weight of 169 metabolic syndrome patients was $74 \pm 10$ (SD) $\mathrm{kg}$. Reduction in 


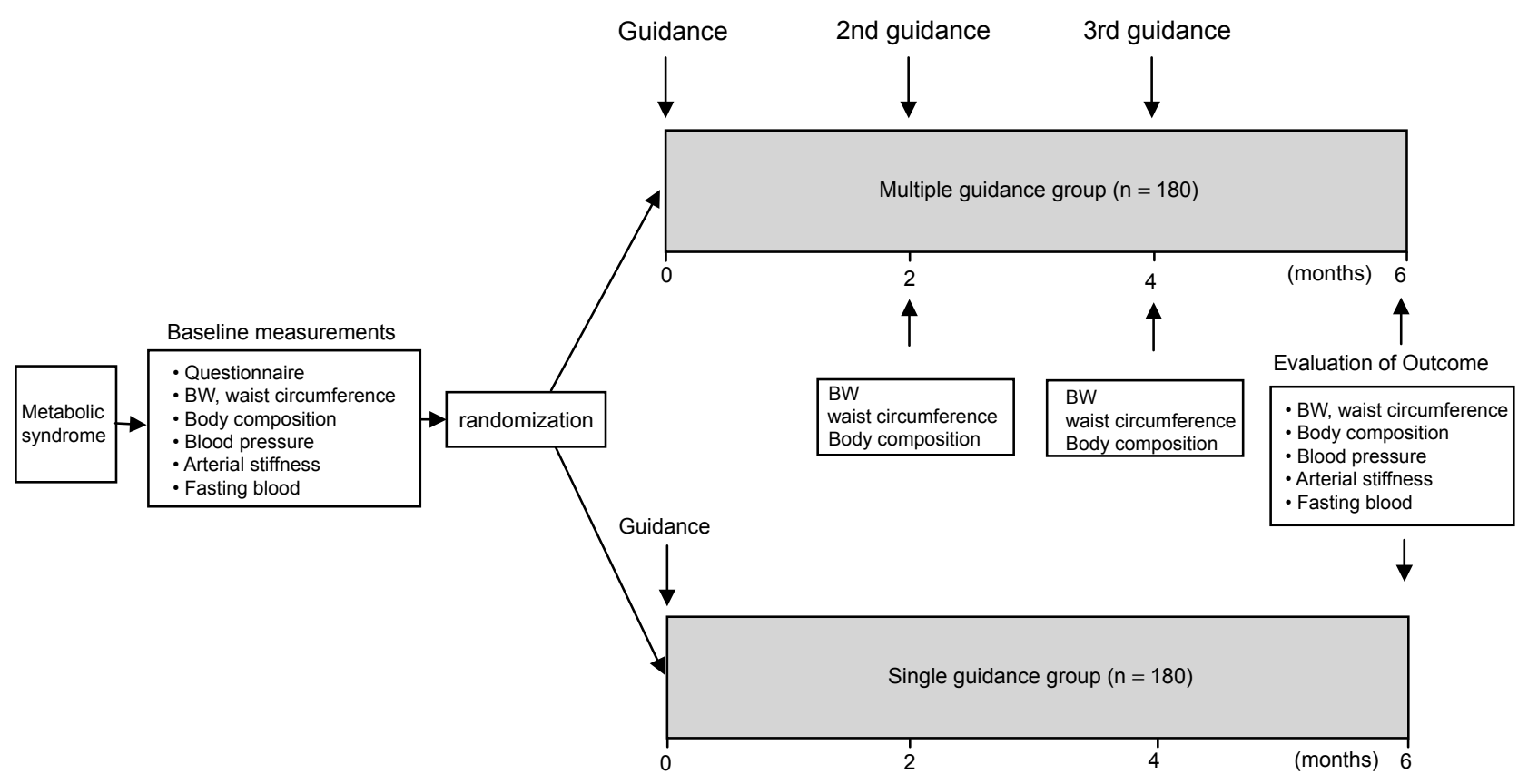

Figure I J-STOP-MetS 2 protocol.

mean body weight following a single guidance session in this group was $1.3 \mathrm{~kg}(1.8 \%)$. If by repeated guidance we could reduce body weight by $5 \%$, the sample size needed to detect the difference between the single and multiple guidance groups is estimated at 180 per group, allowing for a $20 \%$ to $30 \%$ drop out. The diagnostic criteria for the metabolic syndrome in J-STOP-MetS 2 are same as the J-STOP-MetS 1 study, however, only untreated patients are included. Exclusion criteria are also same in both studies. Unmedicated metabolic syndromes are recruited from the patients occasionally found in annual health check up or from outpatients referred for further examination of hypertension, diabetes or dyslipidemia. The advertisement on the recruitment of the examinee is announced in the health promotion lecture for general persons or in the magazine for health promoter of companies.

After written informed consent is obtained, the patients are given the same questionnaire as used in the J-STOPMetS 1 . The subjects visit each center after overnight fast. After the measurements of height, body weight, and waist circumference, blood samples are collected from antecubital vein for determination of glucose and lipid profiles, uric acid concentration, renal and liver functions. In the subjects agree to participate in a substudy some additional blood is withdrawn to measure several adipocytokines such as adiponectin and C-reactive protein, both of them have been reported to be related to atherosclerosis in metabolic syndrome (Haffner 2006; Okamoto et al 2006). Blood pressures are measured by standard mercury sphygmomanometer with the subjects in the sitting position after a rest of at least 5 minutes. The mean of the two measurements is used for the analysis. Heart rate is measured by palpation method. Arterial stiffness as assessed by brachial-ankle pulse wave velocity (baPWV) and ankle-brachial pressure index (ABI) are measured by new device (form PWV/ABI, colin, Japan) in the supine position. Validity, reliability and clinical usefulness of the baPWV and ABI measured by this machine have been reported elsewhere (Cortez-Cooper et al 2003; Munakata et al 2003). Recent study has shown that metabolic syndrome detection status is closely linked to arterial stiffness as assessed by the brachial ankle pulse wave velocity (Tomiyama 2006). So it is of interest if standardized intervention of weight loss could reduce arterial stiffness in metabolic syndrome.

Body composition (fat free mass and fat mass in whole body and at each limb) is measured by eight polar bioelectrical impedance analysis (In body 720, Biospace, Korea). The principle of this measurement has been reported previously (Bedogni et al 2002). Accuracy of this method for the assessment of total and appendicular body composition has been reported in healthy human (Malavolti et al 2003) and peritoneal dialysis patients (Medici et al 2005).

\section{Intervention program}

Three essential components of a weight control program are diet, exercise, and behavioral modifications. Total, 
individualized life style modification is given to all subjects as follows. First of all, the physician explains the pathophysiology of the metabolic syndrome and the risk of future cardiovascular events of this condition. The physician also explains the rationale for advising weight loss and its importance for the maintenance of health. The subjects must be strongly motivated to participate in a weight reduction program by the physicians' introduction.

Then behavioral approach was conducted by a trained nurse to maintain a long-term weight loss. Behavioral therapy involves 1) recognition of unhealthy dietary habits (night meal, between meals, excess alcohol consumption, absence of breakfast, etc), 2) record-keeping of body weight, waist circumference, and physical activity, 3) stimulus control (ie, attention to antecedents of eating that promote excessive intake), 4) cognitive restructuring, and 5) smoking cessation if needed. Tentative goal of weight reduction is set at $5 \%$ of body weight during the next 2 months.

The dietician, then, gives advice on what is an adequate calorie intake to achieve the goal. Weight loss occurs only when energy expenditure exceeds intake. One must know the amount of energy intake keeps the body weight constant. The dietary program, thus, is based on the caloric reduction of about 400 to $600 \mathrm{kcal} /$ day from the value necessary to maintain weight. For example, a kilogram of adipose tissue provides the energy equivalent of $7000 \mathrm{kcal}$ (Munro and Douglas 1983). So, the reduction of 5 kilogram of adipose tissue during 2 months can be achieved by negative calorie intake of $583 \mathrm{kcal} /$ day $(7000 \mathrm{kcal} \times 5 \mathrm{~kg} / 60$ day $)$. The diet also should have the following characteristics: 1) satisfaction of all nutrient needs except energy, 2) practicality and long-term effectiveness (ie, the diet should be both practical for and applicable to the patient's environment, and should be conductive to weight maintenance, and 3) recommendation of salt restriction and DASH food (Sacks et al 2001) for subjects with high blood pressure.

As part of weight-control program, the exercise component is useful and should 1) promote increased energy expenditure, 2) promote fat loss and maintenance of lean body mass, 3) be safe for participating individuals, and 4) promote increases in physical activity levels within the individual's life-style. So, the exercise trainer examines the subjects' physical conditions and conducts an ergometer test to determine the safety level of daily exercise, based on cardiopulmonary responses to exercise. The goal of increase in physical activity is at least 30 minutes per day, 4 to 5 days per week. Exercise intensity is moderate at approximately $40 \%$ to $55 \%$ of heart rate reserve, and consisted primarily of brisk walking.

\section{Randomization}

After the first guidance, subjects are assigned either to a single guidance group or a multiple guidance group (Figure 1). Subjects assigned to a multiple guidance group visit each center every 2 months and receive reevaluation of body weight, weight circumference and body composition. The goal of body weight reduction at next visit in multiple guidance group is a further $5 \%$ reduction of the body weight. If target body weight is not achieved at each visit, the cause is evaluated by the interview of the trained nurse, dietician and exercise trainer, and the adequate advise is presented to the subjects. Subjects assigned to a single guidance group are requested to practice weight reduction program at a rate of $5 \%$ of body weight every 2 month.

\section{Outcome measures}

Outcome measures are body weight, waist circumference, lean body mass, and fat mass, several biochemical markers in the blood (glucose, lipid profiles, liver, and renal functions), and arterial stiffness as assessed by the brachial-ankle pulse wave velocity. Those are studied before and 6 months after the first guidance in all subjects.

This study was approved by the ethic committee of all Rosai Hospitals participated in this study.

\section{Discussion}

A recent Japanese governmental survey has shown that the metabolic syndrome is very prevalent in all adult generations of men and in older women. It is important that greater effort is made to prevent further increases of the metabolic syndrome in the population and to achieve this aim, a public preventive strategy is required.

Excess calorie intake and low energy expenditure can both be causes of the metabolic syndrome. In Japan, westernization of food, which is rich in lipid, and reduced physical activity due to a motorization, are believed to have contributed to an obesity-prone lifestyle (Yazaki and Kadowaki 2006). However, it has been suggested that environmental factors can also significantly influence the development of the metabolic syndrome. For example, Chandola and colleagues (2006) have shown that stress at work is a risk factor for the metabolic syndrome independent of energy intake and physical activity. Furthermore, it has been shown that longer shift work duration is associated with a higher risk of metabolic abnormality (Karlsson et al 2001). In Japan, the frequency of shift work is increasing dramatically (Munakata et al 2001). Moreover, a governmental survey has shown that the frequency of workers who report job stress in 2004 was $61.5 \%$, which is $6.5 \%$ higher than in 1987. The causes of the metabolic syndrome must be 
examined not only from individual dietary and exercise habits but also in the context of environmental factors.

J-STOP-MetS 1 is the largest study undertaken to examine the background of the metabolic syndrome in Japan. The outcome of this study will help identify several unrecognized risk factors for the metabolic syndrome and this information will be used to develop a public preventive strategy aimed at reducing the incidence of the metabolic syndrome in the Japanese population.

The incidence of obesity is increasing in Japan, despite annual health checks. This is possibly because a systematic educational system has been lacking as part of the health check system. Therefore, to effectively reduce morbidity from the metabolic syndrome, we must establish an effective educational system.

From a practical point of view, the frequency of guidance is very important and should be kept to a minimum, to offset absence from work. In diabetes prevention program guidance was given 16 times during 6 months (DPPRG 2002), which is too frequent for workers because they must be absent more than 2 days every month. Also the curriculum was taught by case managers in the diabetes prevention program. In this study a single guidance is delivered by the coordination of highly qualified staff, including a physician, trained nurse, dietician, and exercise professional, in order to improve the quality of guidance and to reduce the total number of guidance sessions needed.

The cutoff value for waist circumference in the diagnosis of the metabolic syndrome is debatable, especially for women. Recent studies have shown that the cutoff value for women, which has been set at $90 \mathrm{~cm}$ for Japanese women, would be more appropriate if shortened to $80 \mathrm{~cm}$ (Miyazaki et al 2005; Hara et al 2006). Although it has been shown that waist circumference corresponding to $100 \mathrm{~cm}^{2}$ of visceral fat area in Japanese women is $92.5 \mathrm{~cm}$ (ECCODJ 2002), the cutoff value must be reduced for small women. For such patients, the risk assessment should be done by assessing the constellation of cardiovascular risks rather than waist circumference alone. Otherwise, a considerable number of pseudo-negative cases will occur. In this study, therefore, we use the Asian criteria for the diagnosis of the metabolic syndrome in women.

It has been shown that compared with usual care, dietary counseling interventions produce modest weight loss, which, however, diminishes over time (Dansinger et al 2007). So it is of particular interest as to how long the effect of our comprehensive weight loss program lasts. To address this issue, we need to add further long-term follow up study.
Finally, the J-STOP-Mets 2 includes several sub-studies which provide scientific merit in their own right. These include the effects of lifestyle modification on arterial stiffness and adipocytokines such as high sensitive Creactive protein and adiponectin. Recently it has been shown that inflammation is involved in the stiffening of the artery (Okamura et al 2004). Because adeponectin has been reported to serve anti-inflammatory action, our study should clarify the mechanisms why visceral fat accumulation increases the arterial stiffness from the view of inflammation.

In conclusion, the J-STOP-MetS is a first large-scale clinical study on the metabolic syndrome in Japan. In 2008, a new law on the management of the metabolic syndrome will be in force. We are obligated to give guidance for lifestyle modifications to all patients diagnosed with the metabolic syndrome at the time of their annual health check. Our study should provide several items of evidences that will be useful for the practical management of the metabolic syndrome and should help with the development of a governmental strategy to reduce morbidity of the metabolic syndrome.

\section{Disclosure}

This study was supported by Grants-in-Aid from the Japan Labor Health and Welfare Organization. The authors have no other conflicts of interest to report

\section{References}

Abe T, Tsutsui H, Namba T. 1972. Studies on the self-rating questionnaire for the screening test of masked depression (SRQ-D) [in Japanese with English abstract]. J Jpn Psychosom Soc, 12:243-7.

Alberti KG, Zimmet P, Shaw J; IDF Epidemiology Task Force Consensus Group. 2005. The metabolic syndrome - a new worldwide definition. Lancet, 366:1059-62.

Baltali M, Gokcel A, Kiziltan HT, et al. 2003. Association between the metabolic syndrome and newly diagnosed coronary artery disease. Diabetes Nutr Metab, 16:169-75.

Bedogni G, Malavolti M, Severi S, et al. 2002. Accuracy of an eight-point tactile-electrode impedance method in the assessment of total body water. Eur J Clin Nut, 56:1143-48.

[CEDSMSJ] Committee to Evaluate Diagnostic Standard for Metabolic Syndrome in Japan. 2005. Nippon Naika Gakkai Zasshi 94:794-809 (in Japanese).

Chandola T, Brunner E, Marmot M. 2006. Chronic stress at work and the metabolic syndrome: prospective study. BMJ, 332:521-5.

Cortez-Cooper MY, Supak JA, Tanaka H. 2003. A new device for automatic measurements of arterial stiffness and ankle-brachial index. Am J Cardiol, 91:1519-22

Dansinger ML, Tastioni A, Wong JB, et al. 2007. Meta-analysis: the effect of dietary counseling for weight loss. Ann Intern Med, 147:41-50.

[DPPRG] Diabetes Prevention Program Research Group. 2002. Reduction in the incidence of type 2 diabetes with lifestyle intervention or metformin N Engl J Med, 346:393-403. 
[ECCODJ] The Examination Committee of Criteria for 'Obesity Disease' in Japan. 2002. New criteria for 'obesity disease' in Japan. Circ J, 66:987-92.

Haffner SM. 2006. The metabolic syndrome: inflammation, diabetes mellitus, and cardiovascular disease. Am J Cardiol, 97(2A):3A-11A.

Hara K, Matsushita Y, Horikoshi M, et al. 2006. A proposal for the cutoff point of waist circumference for the diagnosis of metabolic syndrome in the Japanese population. Diabetes Care, 29:1123-4.

Health Bureau Office of General Affairs Division - Lifestyle Diseases. 2004. National Health and Nutrition Examination Survey Results Overview [online; in Japanese]. Accessed on Dec 3, 2007. URL: http://www. mhlw.go.jp/houdou/2006/05/h0508-1a.html.

Karlsson B, Knutsson A, Lindahl B. 2001. Is there an association between shift work and having a metabolic syndrome? Results from a population based study of 27,485 people. Occup Environ Med, 58:747-52.

Kip KE, Marroquin OC, Kelley DE, et al. 2004. Clinical importance of obesity versus the metabolic syndrome in cardiovascular risk in women: a report from the Women's Ischemia Syndrome Evaluation (WISE) study. Circulation, 109:706-13.

Maeda S. 1985. A study on behavior pattern of patients with coronary heart disease: application of brief questionnaire [in Japanese with English abstract]. Jap J Psychosom Med, 25:297-306.

Malavolti M, Mussi C, Poli M, et al. 2003. Cross-calibration of eight-polar bioelectrical impedance analysis versus dual-energy X-ray absorptiometry for the assessment of total and appendicular body composition in healthy subjects aged 21-82 years. Ann Hum Biol, 30:380-91.

Medici G, Mussi C, Fantuzzi AL, et al. 2005. Accuracy of eight-polar bioelectrical impedance analysis for the assessment of total and appendicular body composition in peritoneal dialysis patients. Eur $J$ Clin Nutr, 59:932-7.

Miyawaki T, Hirata M, Moriyama K, et al. 2005. Metabolic syndrome in Japanese Diagnosed with visceral fat measurement by computed tomography. Proc Japan Acad, 81:1-9
Munakata M, Hiraizumi T, Tomiie T, et al. 1998. Psychobehavioral factors involved in the isolated office hypertension: comparison with stressinduced hypertension. J Hypertens, 16:419-22.

Munakata M, Ichii S, Nunokawa T, et al. 2001. Influence of night shift work on psychologic state and cardiovascular and neuroendocrine responses in healthy nurses. Hypertens Res, 24:25-31.

Munakata M, Ito N, Nunokawa T, et al. 2003. Utility of automated brachial ankle pulse wave velocity measurements in hypertensive patients. $\mathrm{Am}$ J Hypertens, 16:653-7.

Munro JF, Douglas JG. 1983. The management of obesity. Hum Nutr Clin Nutr, 37C:1-19.

Okamoto Y, Kihara S, Funahashi T, et al. 2006. Adiponectin: a key adipocytokine in metabolic syndrome. Clin Sci, 110:267-78.

Okamura T, Moriyama Y, Kadowaki T, et al. 2004. Non-invasive measurement of brachial-ankle pulse wave velocity is associated with serum C-reactive protein but not with alpha-tocopherol in Japanese middle-aged male workers. Hypertens Res, 27:173-80.

Sacks FM, Svetkey LP, Vollmer WM, et al. 2001. DASH-Sodium Collaborative Research Group. Effects on blood pressure of reduced dietary sodium and the Dietary Approaches to Stop Hypertension (DASH) diet. DASH-Sodium Collaborative Research Group. $N$ Engl J Med, 344:3-10.

Sakamoto M. 2006. The situation of the epidemiology and management of obesity in Japan. Int J Vitam Nutr Res, 76:253-6.

Tomiyama H, Hirayama Y, Hashimoto H, et al. 2006. The effects of changes in the metabolic syndrome detection status on arterial stiffening: a prospective study. Hypertens Res, 29:673-8.

Yazaki Y, Kadowaki T. 2006. Combating diabetes and obesity in Japan. Nature Med, 12:73-4.

Yoshiike N, Kaneda F, Takimoto H. 2002. Epidemiology of obesity and public health strategies for its control in Japan. Asia Pac J Clin Nutr, 11(Suppl 8):S727-31. 